\title{
Savoir, pouvoir et inconscient : de la psychanalyse comme dispositif de subjectivation
}

\author{
JEAn-FrançOIS BisSONNETTE
}

Michel Foucault n'a eu de cesse de réinterpréter le sens et la direction de ses recherches. Au cours des différents mouvements qui ont orienté son travail, il est passé d'une archéologie des savoirs, à une généalogie des pouvoirs, pour se consacrer, dans les ultimes développements de sa réflexion, à une analyse des modes de subjectivation hérités de la longue tradition occidentale. En dépit de ces multiples déplacements, je crois que l'on peut déceler une constante dans le fait que Foucault n'ait jamais cessé de se débattre avec un ensemble discursif en particulier, la psychanalyse.

Les quelques commentateurs qui se sont attardés à mesurer l'importance que la psychanalyse a prise dans l'œuvre foucaldienne s'entendent pour souligner son caractère équivoque. Frédéric Gros, par exemple, voit ainsi dans l'attitude de Foucault à l'égard de la psychanalyse une « extraordinaire ambivalence »(Gros 33). Alors que dans l'Histoire de la folie à l'âge classique, Freud est célébré pour avoir renoué, par le biais du langage, avec une expérience de la folie longtemps oblitérée par l'enfermement asilaire, cette appréciation positive se serait retournée en une critique dévastatrice, Freud n'apparaissant plus guère, dans La volonté de savoir, qu'à titre de grand rénovateur de l'archaïque Loi du Père, mise au service, cette fois, de la distinction sociale des classes bourgeoises. 
Si l'on en croit Jacques Derrida, cette ambivalence du rapport à Freud était déjà présente dès l'Histoire de la folie. Le père de la psychanalyse y était dépeint tour à tour comme un «bon » ou un «malin génie », ce statut ambigu empêchant de le localiser avec précision de part ou d'autre de la «coupure épistémologique » censée avoir refermé «l'âge classique » sur luimême. D'un côté, en effet, Freud se voit souvent associé à Nietzsche, Hölderlin ou Artaud, à ceux-là qui ont su se replonger dans cette expérience de la folie où celle-ci révèle son rapport essentiel au langage. De l'autre côté, pourtant, Freud, en ramenant la structure asilaire à son mécanisme fondamental, soit le rapport entre le médecin et son patient, aurait ainsi perpétué l'arraisonnement de la folie par un rationalisme sûr de ses droits. De sorte que, derrière cette ambiguïté d'un Freud rompant avec l'âge classique tout en demeurant son ultime représentant, c'est la cohérence même d'une notion telle que celle d' «épistémè », en tant qu'elle suppose une rupture radicale avec ce qui la précède, qui se trouve alors mise en question.

Comme le fait observer Jacques-Alain Miller, cette «ambivalence affective », qui caractérise, si l'on me permet cette boutade, le rapport de Foucault à Freud, résulte en fait d'une oscillation entre deux fonctions que la psychanalyse occupe dans la démarche foucaldienne. De l'Histoire de la folie jusqu'à Les mots et les choses, elle figure au titre de «principe de l'enquête » (Miller 77). C'est-à-dire que dans ce premier temps, la psychanalyse est envisagée par Foucault comme cette «contre-science » qui, avec l'ethnologie, annonçait la dissolution prochaine de l'homme, entendu comme cet «étrange doublet empirico-transcendantal» (Foucault, Mots 329) dont les sciences humaines ont fait leur fonds de commerce.

Or, dans un second temps, la psychanalyse n'est plus ce qui permet de penser un possible dépassement de l'épistémè moderne; elle est devenue elle-même « objet » de la critique. En cela, elle constitue précisément ce dont il s'agit désormais de s'affranchir pour échapper à la tyrannie 
du désir et des formes de pouvoir qui s'appuient sur son entretien. Cela dit, ce renversement statutaire de la psychanalyse n'aurait pas dissipé l'ambivalence de Foucault à son égard, car c'est en remontant vers la racine de cette créature analytique qu'est l'homme du désir qu'il retrouvera, dans l'érotisme antique, cela même que le dépassement de la psychanalyse devait permettre d'atteindre, soit le libre jeu du plaisir avec lui-même.

Si la psychanalyse peut ainsi traverser, malgré ou justement à cause de cette ambiguité constitutive, l'ensemble de la démarche foucaldienne, cela vient expliciter non seulement certains écueils entre lesquels Foucault chercha à naviguer, mais aussi traduire son positionnement dans le champ intellectuel français, à une époque où la psychanalyse y faisait florès. Didier Eribon, pour sa part, montre qu'en filigrane de son œuvre, Foucault n'a cessé d'entretenir un débat larvé avec Jacques Lacan. Si ces deux penseurs ont bien partagé le postulat d'une dépendance radicale du sujet à l'égard d'un système de signes, c'est dans une sorte de révérence critique à l'égard du psychiatre que Foucault aurait plus tard thématisé l'opposition entre Loi et Norme.

En ce sens, Derrida est peut-être «juste» avec Foucault lorsqu'il lui reproche d'avoir failli à problématiser ses propres "problématisations », et de n'avoir pas su reconnaître que la psychanalyse était de fait la condition de possibilité de sa propre entreprise intellectuelle. Car si Foucault a pu souhaiter que sa critique puisse, comme celle de Deleuze et Guattari, mettre un terme aux prétentions de ceux qu'il appelait «ces pitoyables techniciens du désir» (Foucault, «Préface » 134), le fait que la psychanalyse fasse incessamment retour dans son œuvre et qu'il ne parvienne pas à s'en désempêtrer suggère qu'à travers sa critique, c'est encore la psychanalyse qui parle d'elle-même et qui révèle son propre manque, fécond, d'unité. 
Après avoir rappelé ces quelques commentaires, je voudrais, pour ma part, plutôt que de discuter de l'attitude de Foucault, de son appréciation normative ambivalente ou des difficultés que cela entraîne quant à la cohérence de son projet archéologique, examiner «synchroniquement» le fonctionnement de la psychanalyse, en tant que celui-ci permet d'évaluer la portée heuristique de certains concepts fondamentaux de la réflexion foucaldienne, en particulier ces notions incertaines que sont celles de «dispositif» et de «mode de subjectivation ». Pour ce faire, il me faudra au préalable rappeler les grandes lignes de la généalogie foucaldienne de la psychanalyse de manière à mieux saisir le sens de sa critique. Or, comme je tenterai de le démontrer ensuite, je crois que ce serait sous-estimer la justesse et la portée de cette critique que de n'y voir qu'une condamnation théorico-politique de la psychanalyse. Car la psychanalyse n'est pas si différente de n'importe quel autre discours, puisqu'en elle se trouve posée la question de la vérité. J'essaierai donc de montrer que l'on peut voir à l'œuvre, dans la situation analytique, la dynamique même de ce qui fait le sens du projet foucaldien, soit de définir l'articulation des rapports de pouvoir et de savoir dans la constitution de la subjectivité.

\section{La psychanalyse : genèse et critique d'une « contre-science »}

Dans une conférence portant sur la fonction de l'auteur et à laquelle Jacques Lacan s'était d'ailleurs senti convoqué, Michel Foucault rangea Freud parmi les rares «instaurateurs de discursivité » (Foucault, « Auteur » 805) qui ont fait virer sur ses gonds l'organisation du savoir dans les sociétés modernes. L'affirmation de la dualité du psychisme et de l'importance causale des phénomènes inconscients ayant été, selon Freud, «le premier Schibboleth de la psychanalyse » (Freud 223), ce fut autour de cet énoncé qu'allaient se réorganiser les règles pour 
la production des discours au sujet de la folie, en fonction desquelles un objet tel que l'inconscient put dès lors se laisser connaître. Bien entendu, une telle fondation discursive ne constitua pas pour autant une création ex nihilo. Freud a certainement contribué à changer le regard que l'Occident portait sur la folie, mais cela ne fut possible que par sa participation transformatrice au dispositif intellectuel et institutionnel qui enserrait déjà cette expérience.

C'est dans cette perspective que Freud apparaît comme ce «bon génie » dont parlait Derrida. Si Foucault lui rend hommage, c'est parce que Freud a su arracher la folie à l'emprise violente de la machinerie asilaire. Depuis le «grand renfermement » qui avait jeté pêle-mêle dans les limbes de la déraison tous ceux qui s'écartaient de la morale rationaliste et affairée d'une société bourgeoise en train d'éclore, la folie s'était retrouvée emmurée, réduite au silence et livrée telle une curiosité repoussante du «jardin des espèces » à l'examen peu amène des premiers psychiatres. Là où ceux-ci ne percevaient plus que la mécanique rigoureuse de la maladie mentale, dans le monologue impertinent et évasif de l'insensé, Freud allait découvrir que le moindre symptôme était gorgé de sens, soulignant ainsi que la folie était une expérience qui s'ordonnait tout entière dans le registre du langage.

En ce sens, la découverte de Freud était moins une innovation révolutionnaire que le retour à un type d'expérience de la folie telle que la Renaissance pouvait encore l'éprouver, une expérience que Foucault qualifiait de «tragique », dans la mesure où la folie pouvait y paraître comme un risque inhérent à l'exercice de la raison, voire comme le signe d'une destinée du monde. Freud aura donc réhabilité la parole du fou, en tant que celle-ci recelait une vérité que la «perception asilaire » lui avait déniée. Or, cette vérité du discours du fou n'était plus annonciatrice d'une quelconque prophétie concernant le monde. En dépit de la redécouverte des «pouvoirs du langage » qui allaient «exorcis[er] le phénomène du regard » (Foucault, Histoire 
508) propre à la structure asilaire, dorénavant, la psychanalyse renverrait la parole indéfiniment à elle-même. Ainsi que le montre l'importance pratique et théorique de l'interprétation des rêves, la technique initiée par Freud verse entièrement dans une analyse de la représentation. La vérité que la parole déclamerait désormais, ce ne serait rien de plus que celle du sujet qui l'énonce.

Ce que la psychanalyse découvre dans le jeu des représentations qui encombrent l'esprit du malade, et qui se trahissent jusque dans ses «actes manqués », c'est le battement continu du désir. Elle n'était certes pas la première à lier ainsi la folie à l'emprise des passions, aux fièvres amoureuses et aux dérèglements de la sexualité. Déjà, chez Platon, l’amour était cette « grande ivresse de l'âme », qui en perturbait le fonctionnement. Mais tant qu'elle ouvrait sur une expérience du sublime, cette ivresse n'était pas étrangère à la vérité. Or si, dans son « ingénuité », «la psychanalyse a bien vu que toute folie s'enracine dans quelque sexualité troublée $[\ldots]$ ceci n'a de sens que dans la mesure où notre culture [...] a placé la sexualité sur la ligne de partage de la déraison » (103).

Face à l'amour raisonnable s'ordonnant aux exigences de la bonne société, le sexe et ses vertiges allaient tomber sous une forme de contrainte autrement plus subtile que la loi de la prohibition. Ils allaient dorénavant s'ordonner à la mesure du normal et du pathologique. Or, si « une certaine image de l'homme pivote autour de l'œuvre de Freud », sans que le savoir qui la soutient sorte «pour autant de sa disposition fondamentale » (Foucault, Mots 372), c'est parce que la psychanalyse, en portant à sa limite la liaison de la sexualité et de la maladie, aura fini par dissiper le grand partage entre les fous et les autres. La tyrannie du désir s'impose à tout le monde, et nul ne saurait se targuer d'être parvenu à lui faire définitivement entendre raison. 
Or, en brisant ainsi la logique infâmante du rationalisme triomphant, pour lui substituer le long dégradé de la normalité pathologique, la psychanalyse a conféré une extension sans précédent au domaine des sciences de l'esprit.

Naît alors une grande technologie de la psyché [...] elle fait du sexe à la fois la vérité cachée de la conscience raisonnable et le sens déchiffrable de la folie : leur sens commun, et donc ce qui permet d'avoir prise sur l'une et sur l'autre selon les mêmes modalités (Foucault, « Rapports de pouvoir » 230).

C'est ici que l'appréciation positive initiale finit par se renverser dans la dénonciation des prétentions de la psychanalyse et de ses partisans, qu'il s'agisse des visées adaptatives de Freud faisant le pari d'une réconciliation possible d'un individu clivé par ses conflits inconscients une fois ceux-ci mis au jour, ou bien celles des Reich et consorts, qui prêchèrent la subsomption des luttes prolétariennes sous l'idée d'un affranchissement à l'égard de toutes les contraintes sexuelles. Pas même Lacan n'y échappe, lui qui enferme irrémédiablement le sujet dans sa dépendance constitutive au jeu de la Loi et du Manque, condamnant par avance toute possibilité d'émancipation.

Pour Foucault, cependant, l'échec des visées émancipatoires d'une certaine psychanalyse ne tient pas à la nature insondable et insoluble du désir humain, mais plutôt à l'aveuglement de la tradition freudienne quant à ses propres conditions de possibilité. La psychanalyse appartient pleinement au régime de production de la folie et de la sexualité dont elle feint de critiquer la prétendue logique répressive. Ce n'est pas qu'elle soit elle-même répressive, même si Freud considérait que la répression pulsionnelle était la condition nécessaire de la civilisation, de même que la raison des pathologies qu'elle génère. Freud historicisait déjà la sexualité, tout comme Foucault entreprend de le faire à nouveau. Mais les psychanalystes n’ont pas éclairé leur propre 
inconscient, si l'on peut désigner ainsi la trame généalogique dont ils ont tiré leurs principales méthodes et leurs postulats fondamentaux.

D’abord, malgré sa rupture apparente avec l'ordre asilaire, la psychanalyse en a ramené la rationalité à son expression la plus épurée, à cet « étranglement du rapport entre deux individus » (Foucault, Mots 387) où se produit l'interaction du médecin et du malade. En cela, il y a aussi une parenté évidente de la psychanalyse avec les pratiques confessionnelles du christianisme moyenâgeux. La situation psychanalytique — entendons par cela le face-à-face entre l'analysant et l'analysé - est du même ordre que ces procédures de pouvoir et de savoir inventées par le pastorat chrétien, de manière à faire de l'homme occidental une «bête d'aveu (Foucault, Volonté 80).

La situation psychanalytique est ainsi la mise en scène d'un rapport entre deux subjectivités par le biais de procédures à la fois «juridictionnelles » et « véridictionnelles ${ }^{\mathrm{i}}$, qui se justifient par et à travers une parole soliloque réputée posséder le principe de son propre déchiffrement et donc une efficacité thérapeutique intrinsèque. L'analysé se soumet à une injonction de tout dire, et se trouve ainsi engagé dans la production discursive de lui-même, tandis qu'il fabrique ou «prend conscience » de son intériorité. Procédure véridictionnelle en ce qu'elle vise à produire la vérité du sujet, et par là sa guérison, la situation psychanalytique confirme en même temps l'autorité juridictionnelle de l'analyste, lui qui indique silencieusement la loi qui gouverne le secret des conduites de son vis-à-vis. Si cette autorité a bien un accent mystique portant le malade à «s'aliéner » dans son médecin dans l'espoir de recevoir de lui sa guérison (Foucault, Histoire 528), elle s'authentifie précisément par ce caractère véridique de la parole, où se joue non seulement la prétention à l'objectivité de la science analytique, mais aussi et surtout l'instant décisif de la cure, le « transfert » où la parole délivre enfin la clef de son mal. 
Une lecture rapide de La volonté de savoir peut laisser penser que ce caractère mystificateur ruine la légitimité du savoir et de la pratique analytiques. Pourtant, c'est bien de vérité dont il est question. La situation analytique livre le sujet à une entreprise d'autodécryptage, dans la recherche éperdue de la vérité de son désir. Mais par le fait même, cette vérité, qu'une certaine croyance aux pouvoirs de la raison verrait nimbée d'une lumière émancipatoire, loin d'affranchir l'individu du poids de ses déterminations psychiques, l'attache en fait à lui-même pour mieux l'épingler au tableau des «normalités différentielles » (Foucault, Sécurité 65). Plutôt que de résorber le «malaise dans la culture », la psychanalyse le nomme et, partant, l'approfondit. Dès lors, ce qui s'annonçait au départ comme une ouverture par-delà l'homme que Foucault appelait «l'analytique de la finitude » (Foucault, Mots 323), s'effondre dans le redoublement infini des interprétations où le sujet s'interroge jusqu'à s'enliser.

Il y a donc bel et bien une vérité de la psychanalyse. C'est pour cela qu'elle marche, qu'elle « normalise ». J'aimerais maintenant montrer que si l'on réexamine ce qui vient d'être dit de la situation analytique à travers un prisme conceptuel foucaldien, combinant les notions de « dispositif » et de «mode de subjectivation », l'on pourrait dégager un concept qui permettrait de mieux saisir l'efficacité — au sens d'une « efficacité symbolique » (Lévi-Strauss 205) — de la psychanalyse.

\section{Sur la vérité de l'inconscient : des dispositions affectives?}

Dans La volonté de savoir, la psychanalyse est présentée comme une composante d'un ensemble qui la déborde, baptisé «dispositif de sexualité », opérant la jonction entre cette structure en apparence nouvelle (mais qui, comme le montre la suite de l'Histoire de la sexualité, s'avère poussée de racines bien plus anciennes) et l'immémorial «système d'alliance » 
(Foucault, Volonté 140 sqq.). La critique de la psychanalyse est ce qui permet à Foucault de nommer la rationalité plus large du dispositif de sexualité, qui consiste à mettre le sexe en paroles et à le relier, dans son théorème « juridico-discursif » (110), à la loi de la famille. Or, il me semble que la situation psychanalytique comme telle pourrait très bien être définie comme un « dispositif» à échelle réduite. Il faut bien admettre que le concept de dispositif manque de clarté. Ce qui s'approche le plus d'une définition dans l'œuvre de Foucault consiste en une longue énumération, soit :

un ensemble résolument hétérogène, comportant des discours, des instructions, des aménagements architecturaux, des décisions réglementaires, des lois, des mesures administratives, des énoncés scientifiques, des propositions philosophiques, morales, philanthropiques, bref : du dit, aussi bien que du non-dit [...]. Le dispositif lui-même, c'est le réseau qu'on peut établir entre ces éléments (Foucault, « Jeu » 299).

Il me semble qu'un réseau similaire a pu courir entre le mobilier du père Freud, les écrits de ses divers contempteurs ou partisans, les rêves de l'Homme aux loups et les statuts de la Société internationale de psychanalyse.

La psychanalyse est plus qu'une «discursivité », dans la mesure où elle travaille non seulement l'« esprit» mais aussi les corps. Elle le fait dans un certain espace, au moyen de certaines techniques et avec des résultats empiriquement observables, ce qui est d'ailleurs très contesté. De même, le dispositif s'étend au-delà de l'épistémè, en incluant la part du nondiscursif — dont on peut se demander d'ailleurs si cela ne correspond pas à cet objet ontologiquement ambigu que sont les « choses », en tant qu'elles diffèrent des «mots ». Mais comme l'épistémè, qui est l'ensemble des règles de production et de circulation des énoncés jetant des ponts en travers du fossé qui sépare mots et choses, le dispositif peut être entendu comme un principe de distribution d'éléments divers, alternativement sujets et objets. 
À partir de cette idée d'un dispositif agençant les relations des sujets entre eux de même qu'entre eux et leur environnement physique et social ${ }^{\mathrm{ii}}$, il semble que l'on puisse inférer une articulation conceptuelle avec la notion de «mode d'assujettissement». Foucault signale qu'« il y a deux sens au mot "sujet" : sujet soumis à l'autre par le contrôle et la dépendance, et sujet attaché à sa propre identité par la conscience ou la connaissance de soi. Dans les deux cas, ce mot suggère une forme de pouvoir qui subjugue et assujettit » (Foucault, «Sujet» 1046). Le «mode d'assujettissement» ou de «subjectivation» correspond grosso modo à une forme culturellement transmise de pratiques servant à la problématisation de la subjectivité, qui proposent, comme conduites socialement valorisées, des réponses, qu'elles soient autoritaires ou fruits d'une recherche autonome, à la question de ce qu'est et ce que doit être un sujet. Relève de ce registre l'ensemble de ce que Foucault appelle les «techniques de soi », dont il est à parier qu'il s'en trouve dans toute culture sous une infinité de formes.

Or, la psychanalyse est précisément une de ces techniques. Comme l'étaient la pratique de l'échange épistolaire ou encore la tenue d'un journal intime au temps des stoïciens, la psychanalyse est une technique auto-interprétative, une «herméneutique de soi » (Foucault, «Techniques de soi » 1629). Mais il ne faut pas entendre la notion de «technique de soi »dans une perspective monadique. La subjectivation, la fabrication de la subjectivité est toujours déjà un processus social. Et c'est le mérite de la psychanalyse, qu'elle partage d'ailleurs avec l'ethnologie, d'avoir su montrer que «la chaîne signifiante par quoi se constitue l'expérience unique de l'individu est perpendiculaire au système formel à partir duquel se forment les significations d'une culture » (Foucault, Mots 392).

La psychanalyse s'éclaire ainsi elle-même dans l'importante fonction culturelle qu'elle a été appelée à remplir dans les sociétés occidentales du $\mathrm{Xx}^{\mathrm{e}}$ siècle. Elle a proposé au public 
moderne un service de direction de conscience valant comme technique pour améliorer la maîtrise de soi-même, de même qu'elle a fourni les clefs de la manipulation psychique dont la propagande politique et le marketing commercial ont si bien su user au cours du siècle. En dépit de cette descendance bâtarde, et malgré qu'elle soit elle-même la technique d'un pouvoir valant cher sur le marché de l'affliction, la psychanalyse produit bel et bien de la subjectivité, et il ne fait pas de doute qu'elle a pu souvent aider à réparer bien des sujets brisés, puisque son objectif est d'habiliter l'humain à une certaine autonomie, consciente du poids de ses déterminismes et capable de les sublimer en faisant œuvre utile pour le monde.

La psychanalyse, forme moderne qu'épouse l'antique «souci de soi», demeure-t-elle pour Foucault une «pratique de la liberté » (Foucault, «Éthique du souci de soi » 1527 sqq.)? Pourquoi pas? puisqu'il n'y a pas d'opposition, comme y insiste Foucault, entre pouvoir et liberté. Le sujet n'est pas l'opposé du pouvoir, ni seulement une masse informe sculptée par lui, mais ce qui se produit dans et par les relations de pouvoir, ce qui se construit librement en chaque point de ces relations. Qu'il s'accentue davantage peut-être par sa résistance au pouvoir n'y change rien, sinon qu'il s'agit de la dynamique même de la relation de pouvoir que de susciter et d'inhiber ces résistances. Au demeurant, la résistance est reconnue comme telle pour son rôle important dans la cure analytique, même qu'elle apparaît parfois plus importante que ce qu'elle cherche à dérober au regard de l'analyste. La résistance ne perturbe pas la relation de pouvoir qui sous-tend la situation analytique, ni la production ininterrompue de la vérité du sujet. Au contraire, elle la prolonge, la fait durer, et pointe en même temps vers son propre épuisement cathartique.

En ce sens, la psychanalyse demeure valable dans ses intentions et ses résultats. Il y a bien une vérité de sa vérité, qui fait justement sa valeur thérapeutique. "Le vrai, s'il est dit à 
temps, à qui il faut, et par celui qui en est à la fois le détenteur et le responsable, guérit » (Foucault, Volonté 90). Mais cette vérité exige la croyance. Son effectivité suppose la foi et, en quelque sorte, une certaine part d'aveuglement et de soumission. «Ce qui implique que la psychanalyse opère toujours avec mystification, parce qu'elle ne peut aider personne qui ne croie en elle » (Foucault, « Interview »1485).

En articulant les concepts foucaldiens de dispositif et de mode d'assujettissement, il semble qu'on obtienne une grille d'analyse des rapports liant pouvoir, savoir et subjectivité, qui se superpose parfaitement à la situation psychanalytique. S'il a jamais cherché à le faire, Foucault n'a pas réussi à contourner la problématique freudienne. Celle-ci fait incessamment retour, et Derrida a sans doute raison de dire que c'est la psychanalyse qui s'interroge elle-même dans le texte de Foucault. S'il y a donc une compatibilité des problématiques, le nominalisme de Foucault s'avère néanmoins relativement irréconciliable avec l'affirmation principielle de l'existence de phénomènes psychiques inconscients, entendus comme un invariant humain. En effet, c'est un principe méthodologique pour Foucault que d'user d'« un scepticisme systématique à l'égard de tous les universaux anthropologiques » (Foucault, « Foucault » 1453).

Qu'en est-il, dès lors, de la réalité de l'inconscient? Comment faut-il comprendre l'adéquation des concepts psychanalytiques à la description des phénomènes psychiques? Pour Foucault, tout savoir génère ses objets. Ceux-ci ne préexistent pas au discours qui les nomme, attendant que les lumières de la raison les arrachent à la pénombre de l'inconnu. Or, ces objets n’en sont pas moins réels, même si une « coupure épistémologique » risque un jour de les verser dans l'impensable. L'homme du désir engendré par la psychanalyse doit être considéré comme ce que Foucault nomme une «réalité de transaction», une de ces « figures transactionnelles et 
transitoires qui, pour n'avoir pas existé de tout temps, n'en sont pas moins réelles » (Foucault, Biopolitique 301).

Foucault affirme ainsi dans Surveiller et punir qu'il y a une «généalogie » à faire de «1"“âme" moderne ». «Il ne faudrait pas dire que l'âme est une illusion, ou un effet idéologique. Mais bien qu'elle existe, qu'elle a une réalité, qu'elle est produite en permanence ». Par-delà les « concepts divers » qui la nomment, cette âme, cette psyché serait « le corrélatif actuel d'une certaine technologie de pouvoir» (Foucault, Surveiller 38). Or, cette technologie, la psychanalyse en a élaboré plusieurs des propositions fondamentales.

On ne peut donc pas dire que le «désir » soit une fausseté, même si Foucault considère que l'attention qui lui est portée détourne et nuit à l'expressivité d'un érotisme affranchi de toute fixation sur des substrats sexués. D’ailleurs, le désir demeure toujours comme un certain « audelà du principe de plaisir », intimement lié au fonctionnement des rapports de pouvoir qui visent à le cerner et à le cultiver. Ce me semble bien être ce que veut dire Foucault lorsqu'il parle du plaisir de l'aveu, qui rend désirable l'assujettissement au pouvoir qui le requiert.

L'extension indéfinie du dispositif de sexualité n'est donc pas le résultat d'une machinerie monstrueuse et impersonnelle, c'est plutôt qu'elle s'entretient elle-même en générant une plus-value de plaisir à la production de sujets désirants. D'où le fait qu'il a pu être à la mode à une certaine époque de consulter un psychanalyste. D'où ce curieux effet de vérité qu'est aussi l'accroissement des symptômes aux abords de la résolution finale de la cure psychanalytique, comme si le sujet, par sa résistance, cherchait à étirer et à maximiser le plaisir de l'analyse. Ainsi, « le plaisir découvert reflue vers le pouvoir qui le cerne. » Et de l'analysé à l'analysant, au cœur de cette expérience ambiguë du «transfert » et du «contre-transfert », s'échangent les coups de ce jeu de « captation et séduction », d' « affrontement et renforcement réciproque », qui 
lie invinciblement l'expérience du plaisir à ce dispositif de savoir et de pouvoir (Foucault, Volonté 61 sq.).

La question se pose alors d'imaginer comment il serait possible de concevoir la dimension psychique des processus de subjectivation tels qu'ils se produisent en vertu de la rationalité des dispositifs de pouvoir et de savoir, et tels qu'ils s'expriment par diverses «conduites » relativement indéterminées. Ici, nous sortons de l'orthodoxie foucaldienne pour risquer un concept nouveau. Ne pourrait-on pas parler de quelque chose comme des « dispositions affectives », pour caractériser cet effet des dispositifs de pouvoir et de savoir, dont une part de l'efficacité consisterait non seulement à «conduire des conduites», mais aussi à façonner des expériences affectives qui en formeraient le soubassement ${ }^{\mathrm{iii}}$ ? Cela voudrait aussi dire que l'inconscient serait non seulement «structuré comme un langage », ainsi que l'estimait Lacan, mais structuré a fortiori par son inscription dans des dispositifs de savoir et de pouvoir ayant leur propre historicité. La psychanalyse serait donc, quant à elle, une technique visant à agir sur de telles dispositions.

En soulignant que l'on se trouverait par là reconduit, par une sorte d'éternel retour, à la question phénoménologique du sujet faisant l'expérience du monde, ce de quoi Foucault cherchait justement à émanciper la pensée pour y substituer plutôt le projet d'une « ontologie historique de nous-mêmes » (Foucault, «Lumières » 1393), cette modeste proposition me paraît ouvrir sur un vaste horizon de recherches, celui d'une histoire de l'affectivité. 


\section{Notes}

${ }^{1}$ Foucault traita de ce rapport entre juridiction et véridiction ainsi que de l'idée d'un « droit de la vérité » dans son cours de 1978-1979, Naissance de la biopolitique (37).

2 Des relations de pouvoir et de savoir inscrites dans la matérialité : songeons à l'efficacité purement formelle du dispositif architectural panoptique, et à son utilité politique.

${ }^{3}$ C'est Jacques Donzelot qui a, le premier, indiqué cette direction, écrivant dans « Le savoir qui fonde le pouvoir »: «Pour comprendre l'ordre et le désordre actuels, mieux vaut essayer de comprendre les "dispositions" exigées de l'individu» (X). Or, il en va pour lui de «compétences» telles que la «capacité d'initiative» et «l'engagement personnel», et non d'affectivité. Je remercie par ailleurs M. Christophe Perrin pour avoir attiré mon attention sur le fait que l'expression «disposition affective » est ce par quoi a été traduit en français le concept de «Stimmung » forgé par Martin Heidegger. Sachant que Foucault a reconnu sa dette envers le penseur allemand ( Tout mon devenir philosophique a été déterminé par ma lecture de Heidegger » [Foucault, «Retour de la morale » 1522]), il y a lieu de croire que l'usage que je propose d'une telle notion s'avère compatible avec la démarche intellectuelle de Foucault.

\section{Ouvrages cités}

Derrida, Jacques, "“Être juste avec Freud" », dans Résistances de la psychanalyse, Paris, Galilée, 1996, p. 89-146.

Donzelot, Jacques, «Le savoir qui fonde le pouvoir », Libération, 19 et 20 juin 2004, p. X.

Eribon, Didier, Michel Foucault et ses contemporains, Paris, Fayard, 1994, p. 233-263.

Foucault, Michel, «L'éthique du souci de soi comme pratique de la liberté », dans Dits et Écrits II. 1976-1988, Paris, Gallimard, 2004, p. 1527-1548.

—, «Foucault », dans Dits et écrits II. 1976-1988, Paris, Gallimard, 2004, p. 1450-1455.

_-, Histoire de la folie à l'âge classique, Paris, Gallimard, 1972.

—, «Interview de Michel Foucault », dans Dits et écrits II. 1976-1988, Paris, Gallimard, 2004, p. $1475-1486$.

—, «Le jeu de Michel Foucault », dans Dits et écrits II. 1976-1988, Paris, Gallimard, 2004, p. 298-329.

—, Les mots et les choses, Paris, Gallimard, 1966. 
-, Naissance de la biopolitique. Cours au Collège de France 1978-1979, Paris, Gallimard/Seuil, 2004.

—, «Préface », dans Dits et écrits II. 1976-1988, Paris, Gallimard, 2004, p. 133-136.

—, «Qu'est-ce que les Lumières? », dans Dits et écrits II. 1976-1988, Paris, Gallimard, 2004, p. 1381-1397.

—, «Qu'est-ce qu'un auteur? », dans Dits et écrits I. 1954-1969, Paris, Gallimard, 1994, p. 789821.

—, «Les rapports de pouvoir passent à l'intérieur des corps », dans Dits et écrits II. 1976-1988, p. 228-236.

—, «Le retour de la morale », dans Dits et écrits II. 1976-1988, Paris, Gallimard, 2004, p. 15151526.

- Sécurité, territoire, population. Cours au Collège de France 1977-1978, Paris, Gallimard/Seuil, 2004.

—, «Le sujet et le pouvoir », dans Dits et écrits II. 1976-1988, Paris, Gallimard, 2004, p. 10411062.

—, Surveiller et punir, Paris, Gallimard, 1975.

—, «Les techniques de soi », dans Dits et écrits II. 1976-1988, Paris, Gallimard, 2004, p. 16021632.

—, La volonté de savoir (Histoire de la sexualité I), Paris, Gallimard, 1976.

Freud, Sigmund, «Le Moi et le Ça », dans Essais de psychanalyse, Paris, Petite Bibliothèque Payot, 1982, p. 219-275.

Gros, Frédéric, «Foucault penseur de la psychanalyse, dans l'Histoire de la folie et La volonté de savoir », La célibataire, automne 2004, p. 33-54.

Lévi-Strauss, Claude, Anthropologie structurale, Paris, Plon, 1958.

Miller, Jacques-Alain, «Michel Foucault et la psychanalyse », dans Michel Foucault philosophe. Rencontre internationale, Paris 9, 10, 11 janvier 1988, Paris, Seuil, 1989, p.77-84. Édition Gallimard (Paris) : p. 228-236. 